\title{
EMISSIVITY OF FROZEN REGIONS RETRIEVED FROM AQUARIUS MEASUREMENTS
}

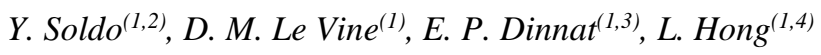 \\ (1) NASA Goddard Space Flight Center, Greenbelt, MD, USA \\ (2) Universities Space Research Association, Columbia, MD, USA \\ (3) Chapman University, Orange, CA, USA \\ (4) Science Applications International Corporation, McLean, VA, USA
}

\begin{abstract}
The land emissivity model used in the Aquarius data processing has been updated for the latest data release (V5.0). In order to improve the estimates of the brightness temperatures of frozen regions, the new model uses values of surface emissivity that have been estimated from the Aquarius measurements averaged over the entire duration of the mission. The retrieved emissivities depend on the geographic location, but they depend only marginally on time, temperature and snow cover.
\end{abstract}

Index Terms- Aquarius, frozen soil, modeling

\section{INTRODUCTION}

The land emissivity model used by the Aquarius mission to estimate the antenna temperatures over land was updated for the latest data release ([1], Appendix B). This update addressed several issues in the previous land model (e.g. the presence of discontinuities in the time series of expected antenna temperatures in an orbit) and improved the consistency between the land models used by the Aquarius and the Soil Moisture Active/Passive (SMAP) mission [2]. One of the goals was to improve the estimated brightness temperatures of frozen regions.

In the previous land model ([3], Appendix B) land was considered frozen if the surface temperature was below zero. In that case, the surface was modelled as a homogeneous halfspace with the dielectric constant of snow from [4] (section E-6.1) and the emissivity was computed using this dielectric constant in Fresnel's equations for reflectivity.

Although there are other models for the dielectric constants of frozen surfaces, dry conditions correspond to large wave penetration depths, which make it difficult to model the surface emissivity.

The new land model uses a different approach: it considers the actual measurements made over frozen regions and uses them to retrieve the emissivities. This contribution describes this new approach and presents some of its results.

\section{METHOD}

In the updated model, the emissivity when the ground is frozen (defined below) is obtained by definition as:

$$
E=\frac{T B}{T}
$$

where $T B$ is the surface brightness temperature measured by Aquarius, $T$ is the thermodynamical temperature of the surface, and $E$ is the emissivity. Where the scene observed by the instrument is not entirely made up by frozen land, Eq. 1 needs to be modified to account for the contributions of other types of surfaces and becomes:

$$
E_{\text {frozen }}=\frac{T B-\sum_{i} E_{i} T_{i} f_{i}}{T_{\text {frozen }} f_{\text {frozen }}}
$$

where the summation is made on the different types of surface (water, sea ice, non-frozen land) and $f$ denotes the fraction of each type of surface within the footprint of the instrument. In Eq. 2, the emissivities of the other types of surfaces are computed as in Appendix B of [1].

The emissivities of frozen regions were retrieved using Eq. 2 for the entire Aquarius dataset, i.e., from August $25^{\text {th }}, 2011$, to June $7^{\text {th }}, 2015$, whenever all the following conditions were met:

- The surface temperature is lower than $-2{ }^{\circ} \mathrm{C}$ (avoids thawed soil);

- The percentage of land is higher than $99 \%$ (avoids coastlines); 


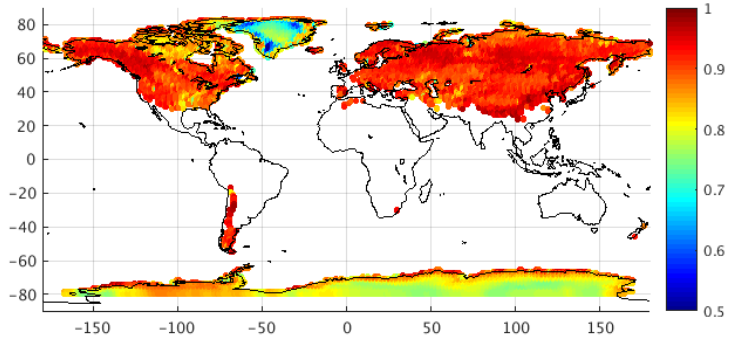

Figure 1: Retrieved emissivities using the Aquarius inner beam and horizontal polarization

- The retrieval of the surface brightness temperature was successful.

The retrieved emissivities have then been binned (i.e., drop in a bucket averages) into a $1 \mathrm{x} 1$ degree grid.

Since emissivities depend on the incidence angle and on the polarization, the binning was done separately for each of the six Aquarius channels (three incidence angles and two polarizations) [6].

\section{RESULTS}

The results of this approach are six latitudelongitude tables with the average retrieved emissivities. Figure 1 shows one of these maps for the inner beam (beam 1) and horizontal polarization.

The blank spaces correspond to cells where the criteria in section 2 did not occur often enough to obtain a meaningful average (i.e., less than 5 valid observations).

\subsection{Comparison with values used by SMOS and Aquarius}

Previous studies have provided estimates of the dielectric constant of frozen soils, ice and snow. Some of these values have been used operationally by the SMOS mission [7] (i.e., frozen soil from [8] and ice from [9]), and by the Aquarius mission [3] (i.e., snow from [4], section E-6.1). We have compared the emissivities obtained from these values and the Fresnel's equations against the emissivities retrieved by this method.

Figure 2 shows that none of the theoretical values matches well the measurements for all channels. Also, the use of only one fixed value would be ill-suited to represent the spatial variations in Figure 1.

\subsection{Temporal stability}
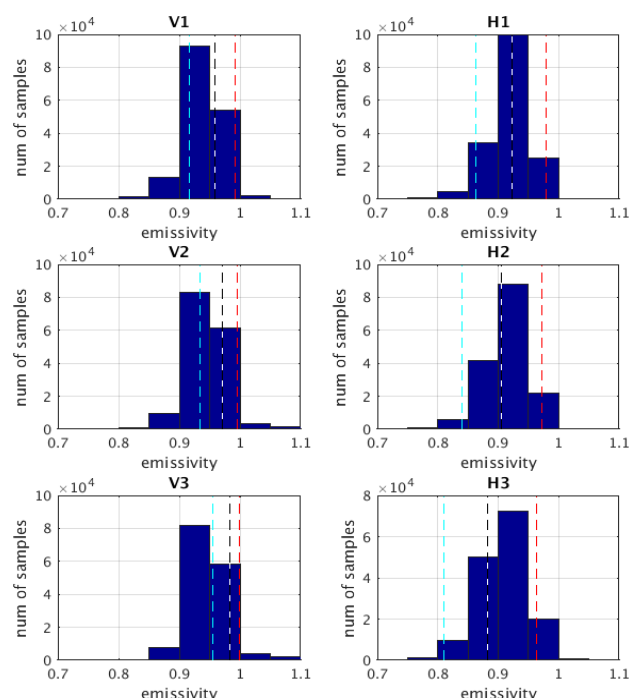

from AQ measurements - Aquarius (snow)

- - SMOS (ice)

SMOS (frozen soil)

Figure 2: Histograms of the retrieved emissivity over North America, Europe and Asia (January 2012) for all the Aquarius channels. (Greenland is excluded)

The retrieved emissivities vary with the geographic location. However, for a particular location, the retrieved emissivities are quite stable. For every month of the Aquarius dataset, we binned the retrieved emissivities to form maps similar to Figure 1. Then, for each grid cell, we computed the standard deviation between different months. The standard deviations for each cell are shown in Figure 3.

The high standard deviations in Greenland appear to be associated with an unusual melt event that occurred in 2012 [10]. The isolated red dots in China and in the Andes correspond to grid cells where few points were used to compute the standard deviation.

\subsection{Effect of snow cover}

Frozen regions are often covered in snow. Therefore, the retrieved emissivities might depend on the amount of accumulated snow at the time of the measurements. Figure 4 shows, for every channel, the retrieved emissivities as a function of the Snow Water Equivalent (SWE), as predicted by the NCEP (National Centers for Environmental Prediction) GFS (Global Forecast System) model. The color is proportional to the density of measurements. In every plot, is also indicated the straight line that best fits the data along with its equation.

The emissivities for vertical polarization show little to no variation with the SWE. Values for 


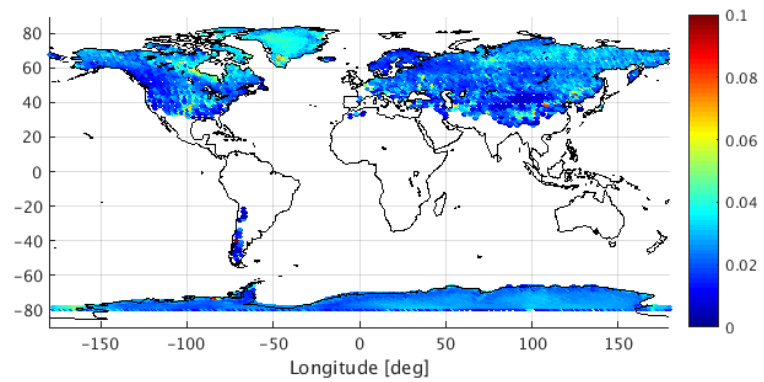

Figure 3: Map of the standard deviations of retrieved emissivities

the horizontal polarization appear to be more dependent on the SWE, consistently with the findings of previous studies, e.g. [11]. Higher incidence angles also appear to be more dependent on the SWE, for the horizontal polarization.

\section{CONCLUSIONS}

This study considered a new approach to improve the estimates of brightness temperatures of frozen regions. This approach uses the surface emissivities retrieved from the actual measurements. The retrieved emissivities showed spatial variations, but were mostly stable over time. Also, the effect of the snow layer covering the frozen soil appears to be small, especially in vertical polarization or at low incidence angles.

This approach was developed for the Aquarius mission at a time when the mission was no longer operational. However, given the stability in time of the retrieved emissivities, it would be reasonable to implement the same approach in an operational mission, after a sufficient amount of data (at least one year) has been collected, and frozen soil in different parts of the globe has been observed.

\section{REFERENCES}

[1] Aquarius Project: Algorithm Theoretical Basis Document, Aquarius Salinity Retrieval Algorithm, version 5, appendix B, 2017.

[2] SMAP Project: Algorithm Theoretical Basis Document, SMAP L2 and L3 Radiometer Soil Moisture (Passive), version 4.0, 2012.

[3] Aquarius Project: Algorithm Theoretical Basis Document, Aquarius Salinity Retrieval Algorithm, version 2, appendix B, 2012.

[4] F. T. Ulaby, R. K. Moore and A. K. Fung, Microwave Remote Sensing: Active and Passive,
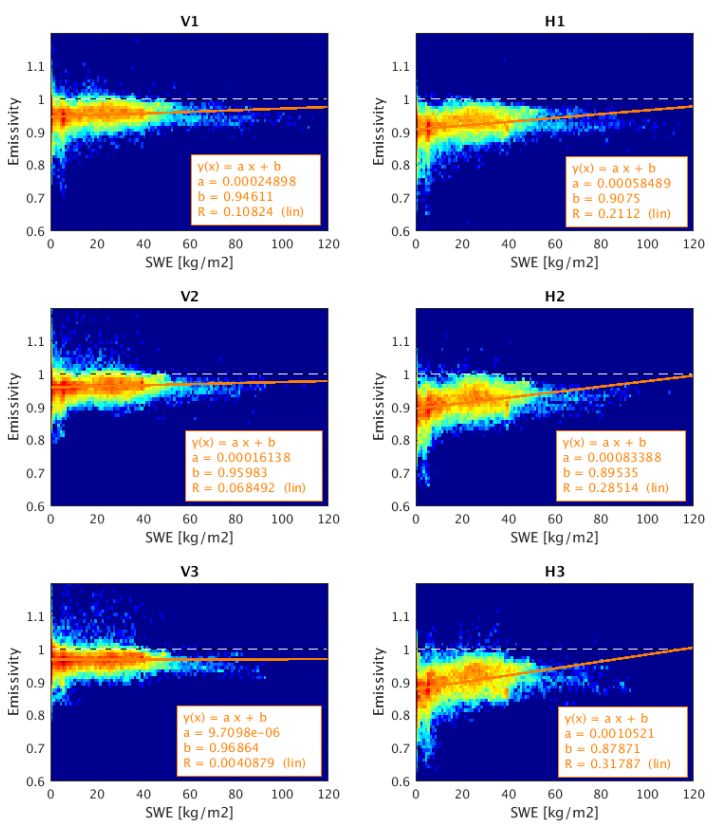

Figure 4: Retrieved emissivities for the different Aquarius channels as a function of the Snow Water Equivalent (SWE)

Volume 3: from theory to applications, Artech Inc., Norwood, 1986

[5] M. T. Hallikainen, F. T. Ulaby, M. C. Dobson, M. A. El-Rayes and L. K. Wu, "Microwave dielectric behavior of wet soil-part 1: Empirical models and experimental observations," IEEE Transactions on Geoscience and Remote Sensing, vol. 1, pp.25-34, 1985.

[6] D. M. Le Vine, G. S. Lagerloef, F. R. Colomb, S. H. Yueh and F. A. Pellerano, "Aquarius: An instrument to monitor sea surface salinity from space," IEEE Transactions on Geoscience and Remote Sensing, vol. 45, n. 7, pp.2040-2050, 2007.

[7] SMOS Project: Algorithm Theoretical Basis Document (ATBD) for the SMOS Level 2 Soil Moisture Processor Development Continuation Project, issue 3.10, section 3.1.4.2, 2017.

[8] M. T. Hallikainen, "Retrieval of snow water equivalent from NIMBUS-7 SMMR data - effect of land-cover categories and weather conditions," IEEE Journal of Oceanic Engineering, vol. 9, pp. 372-376, 1984.

[9] C. Matzler and U. Wegmuller, "DielectricProperties of Fresh-Water Ice at MicrowaveFrequencies," Journal of Physics D-Applied Physics, vol. 20, pp. 1623-1630, 1987.

[10] L. Brucker, E. P. Dinnat and L.S. Koenig, "Weekly gridded Aquarius L-band radiometer/scatterometer observations and salinity 
retrievals over the polar regions-Part 2: Initial product analysis", The Cryosphere, vol. 8, 915-930, 2014

[11] N. Maass, L. Kaleschke, X. Tian-Kunze and M. Drusch, "Snow thickness retrieval over thick Arctic sea ice using SMOS satellite data," The Cryosphere, vol. 7, n. 6, pp.1971-1989, 2013. 